\title{
Enfoque clínico y diagnóstico del absceso hepático
}

\author{
Análida Elizabeth Pinilla $\mathbf{R}^{1}$, Myriam Consuelo López $\mathbf{P}^{2 a}$, \\ Blanca Castillo $M^{1 a}$, Martha Isabel Murcia $A^{3 a}$, \\ Rubén Santiago Nicholls $0^{2,4}$, Sofía D uque $B^{2,4 b}$, \\ Luis Carlos 0 rozco V5.
}

\section{A diagnostic approach to hepatic abscess}

Background: The non invasive diagnosis of amebic liver abscess allows the use of empirical therapy without the requirement of invasive diagnostic procedures. Aim: To determine the discriminatory capacity of clinical, laboratory and ultrasound studies for the etiological diagnosis of liver abscess. Patients and methods: Sixty one patients were initially included in this prospective study, but 12 did not comply with the inclusion criteria. Of the rest, 29 (59\%) had an amebic liver abscess, 16 (33\%) had a pyogenic liver abscess and four (8\%) had an abscess of mixed etiology. Blood cultures were done in 42 patients. Ultrasound guided needle aspiration was done in 7 patients with amebic liver abscess and 13 patients with non amebic liver abscess. Results: The clinical picture and ultrasound fndings were similar in all types of amebic abscess. ELISA test for IgG anti-Entamoeba histolytica antibodies were positive in $100 \%$ of patients with amebic liver abscess. Antibodies measured by gel diffusion were positive in $93 \%$. All patients with mixed liver abscess had positive antibodies and some of them positive culture. Blood cultures were positive for anaerobic bacteria in five patients. Cultures of aspirated material were positive in 7 patients (obligate anaerobic bacteria in 3 and facultative anaerobic bacteria in the rest). The most common complications, whatever the etiology, were right pleural effusion and systemic inflammatory response. Conclusions: A final model of binomial regression analysis revealed that age under 40 years, an hematocrit greater than $35 \%$ and an elevation in prothrombin time of less than 1.5 seconds had enough discriminatory capacity for the diagnosis of amoebic liver abscess (Rev Méd Chile 2003; 131: 1411-20).

(Key Words: Amebiasis; Amebic; Liver Abscess)

Recibido el 8 de julio, 2002. Aceptado en versión corregida el 23 de septiembre, 2003. Financiación: División de Investigación Sede Bogotá. Universidad Nacional de Colombia, proyectos № 709150- 809159.

${ }^{1}$ Departamento de Medicina Interna. Universidad Nacional de Colombia, Bogotá. ${ }^{2}$ Departamento de Salud Pública y Tropical, Instituto de Salud Pública, Universidad Nacional de Colombia. ${ }^{3}$ Departamento de Microbiología, Universidad Nacional de Colombia. ${ }^{4}$ Instituto Nacional de Salud, Bogotá, Colombia. ${ }^{5}$ Escuela de Enfermería, Universidad Industrial de Santander, Colombia. ${ }^{\text {Bacterióloga. }}{ }^{\text {bBóloga }}$

Correspondencia a: Análida Elizabeth Pinilla R. Departamento de Medicina Interna, Universidad Nacional de Colombia, Bogotá. Telefax 57-1-3165000, extensión 15167.

E mail: apinilla@supercabletv.net.co; apinillaroa@hotmail.com 
L a amebiasis es una enfermedad producida por Entamoeba histolytica de distribución mundial. Se estima que infecta alrededor de 500 millones de personas anualmente y que de ellas 110.000 mueren por complicaciones causadas por este agente. El 1\% de las personas infectadas pueden desarrollar patologías potencialmente fatales como la colitis amebiana fulminante 0 el absceso hepático amebiano (AHA) ${ }^{1}$. El AHA es resultado de la invasión al hígado por E histolytica desde un foco intestinal; en la mayoría de los casos existe sólo un absceso localizado generalmente en el lóbulo hepático derecho, el cual recibe la mayor parte de la circulación portal. La frecuencia de AHA es 3 veces mayor en hombres que en mujeres ${ }^{2}$.

$\mathrm{Si}$ se aplican en Colombia las tasas de prevalencia de amebiasis reportadas en la Encuesta Nacional de Morbilidad de 1980, se encuentra que aproximadamente 3.025 .000 personas son portadoras asintomáticas de E histolytica y que 1.075.000 han sufrido algún tipo de enfermedad amebiana intestinal 0 extraintestinal ${ }^{3}$. Sin embargo, en el estudio de 140 muestras realizado por Guzmán y cols para diferenciar E histolytica de E dispar mediante la determinación de adhesina para $\mathrm{E}$ histolytica en materia fecal, se encontró una prevalencia del complejo E histolytica/E dispar 16,42\% y de 1,43\% para E histolytica al extrapolar los resultados de acuerdo a la población de la encuesta nacional de morbilidad de 19803,4.

No obstante, para el diagnóstico de absceso hepático (AH) se debe tener en cuenta que puede ser de origen amebiano o no amebiano. Si se sospecha clínicamente, se requiere de exámenes imaginológicos (el más utilizado es el ultrasonido), inmunológicos y microbiológicos para confirmar la etiología. Sin embargo, la demostración del agente etiológico, en el caso del AHA es de riesgo para el paciente, ya que requiere de métodos invasivos como la punción hepática. En muchos casos no logra el hallazgo del parásito, conllevando al empleo de terapia empírica con metronidazol y otros antibióticos sin poder diferenciar el AHA del absceso hepático piógeno (AHNA).

Debido a las dificultades mencionadas, las pruebas inmunodiagnósticas para detectar anticuerpos contra E histolytica han sido de gran utilidad en casos de amebiasis extraintestinal. Se han descrito las técnicas de hemoaglutinación indirecta ${ }^{5}$, inmunodifusión (ID) ${ }^{6}$, fijación del com- plemento $^{7}$, contrainmuno-electroforesis ${ }^{8}$, inmunofluorescencia indirecta ${ }^{9}$ y ELSA $^{10}$. Por otra parte, el Western-Blot (WB), permite la detección de antígenos específicos de E histolytica, los cuales son reconocidos por sueros de individuos sintomáticos y asintomáticos evitando las reacciones cruzadas con otros parásitos intestinales ${ }^{11}$.

En casos de AHNA se ha encontrado que la fiebre es un hallazgo típico y los agentes que se han aislado con mayor frecuencia son Klebsiella pneumoniae y Escherichia coli. Algunos autores han reportado $55 \%$ de hemocultivos positivos y hasta $73 \%$ de cultivos positivos del pus ${ }^{12}$. También se han reportado casos de Mycobacterium tuberculosus y de Enterococcus faecalis ${ }^{13}$. En la investigación de Pinilla y cols se informó que el diagnóstico de $\mathrm{AH}$ se realizaba de acuerdo a la historia clínica y la concordancia entre las pruebas inmunodiagnósticas en AHA y las pautas para el diagnóstico diferencial del AHA y del AHNA ${ }^{14}$.

\section{Material y MÉTOdO}

Se realizó un estudio prospectivo de corte transver$\mathrm{sal}^{15}$, de evaluación de tecnologías diagnósticas, la prueba de referencia para el diagnóstico de $\mathrm{E}$ histolytica fue el ELISA ${ }^{10}$. Se tomó una población de sujetos con AH en el Hospital San Juan de Dios de Bogotá, analizados desde marzo de 1996 hasta diciembre de 1999. Se estudiaron 61 pacientes con diagnóstico inicial de $\mathrm{AH}$ por cuadro clínico e imaginológico. Los criterios de exclusión fuemon: serología positiva para VIH al ingreso, diagnóstico de cualquier tipo de cáncer con tratamiento inmunosupresor, ingestión de algún corticoide durante el último mes, terapia antibiótica por más de $72 \mathrm{~h}$ antes de la hospitalización y la negativa a ingresar en el estudio. La investigación fue aprobada por la División de Investigación y el Comité de Auditonáa Médica del Hospital San Juan de Dios de Bogotá, todos los pacientes firmaron el consentimiento informado.

Una vez realizada la encuesta que incluía variables sociodemográficas, examen físico, complicaciones y tratamiento, exámenes de laboratorio: cuadro hemático, aminotransferasas, fosfatasa alcalina, bilimubinas, tiempo de protrombina (TP), hemocultivos, ELSA, ID, coprológico, radiografía del tórax y ecografía hepática. En caso de tener pruebas inmunodiagnósticas negativas para E histolytica se consi- 
deró el diagnóstico de AHNA y se procedió a la punción percutánea dirigida por ecografía.

Para el inmunodiagnóstico se tomaron $2 \mathrm{ml}$ de sangre y se centrifugaron a $250 \mathrm{~g}$ durante $5 \mathrm{~min}$. Se separó el suero de la muestra, se distribuyó en alícuotas y se almacenó a $-20^{\circ} \mathrm{C}$. Se siguió la técnica de ID y ELISA según Nicholls ${ }^{10}$. Se recolectaron tres hemocultivos y tres cultivos del aspirado del drenaje para gérmenes aerobios y anaerobios (Sistema BacT/Alert FAN Aerobic y Anaerobic). Los gérmenes comunes se identificaron mediante pruebas bioquímicas tradicionales $\mathrm{y}$ los anaerobios mediante el sistema Minitek ${ }^{16}$.

A cada variable se le calculó el área bajo la curva del receptor operador (AROC) con sus intervalos de confianza (IC) de $95 \%{ }^{17}$ y en el caso de las variables continuas se seleccionaron los puntos de corte que dieran una mayor discriminación y cuyo límite inferior de las AROC fuera $\geq 0,5$. Debido a la alta prevalencia de AHA 0,67 IC 95\% $(0,52-0,80)$ se utilizó la regresión binomial ${ }^{18}$, la cual permite calcular las probabilidades de las diferentes combinaciones de variables con las cuales se realizaron los análisis de las AROC.

Para el análisis sólo se incluyeron las variables que en la tabla tetracónica presentaron marginales $\geq 10^{19}$. Después se realizó un análisis multivariado con la regresión binomial para obtener AROC de las diferentes combinaciones de las variables. Se utilizaron IC de 95\% en todos los análisis; para los IC de las AROC se utilizó la binomial exacta y para compararlas se utilizó el algoritmo sugerido por Delong ${ }^{20}$. El análisis se realizó con Stata Release 6,021.

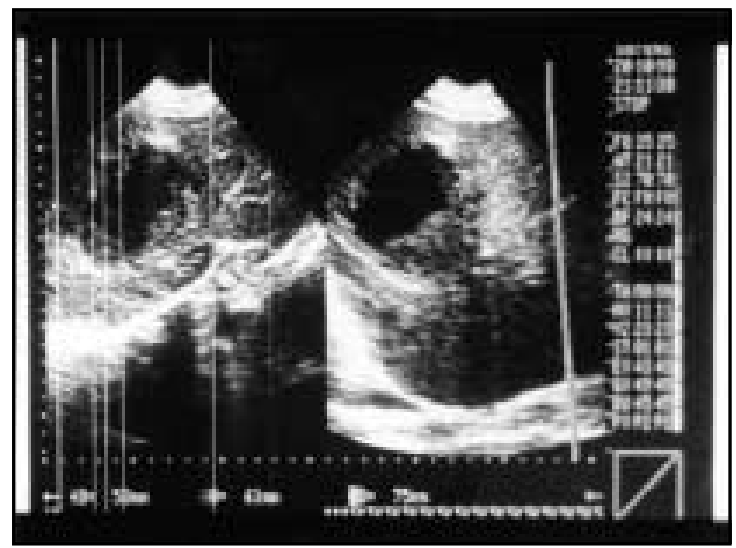

FiguRa 1. Ecografía hepática que muestra absceso hepático único.

\section{RESULTADOS}

Se recolectaron 61 casos de AH, durante 4 años, se excluyeron 12 por diferentes causas: terapia antibiótica previa, corticoides de larga data, ecografía hepática falsa positiva, Mycobacterium tuberculosus, VIH, quiste hepático o no aceptación del paciente. Los 49 casos evaluados se distribuyeron así: 29/49 (59\%) AHA, 16/49 (33\%) AHNA y 4/49 (8\%) absceso hepático mixto (AHM). El promedio de edad de los pacientes con AHA y AHNA fue de 36 y 45 años, mientras que la relación hombremujer fue de $24 / 5$ y 11/5 respectivamente.

La clínica fue similar sin importar la etiología excepto por la presencia de dolor pleurítico en la base del hemitórax derecho para AHA y hepatomegalia con prolongación del TP en AHNA. Se realizó ecografía hepática a todos los pacientes (Figura 1). La radiografía del tórax mostró elevación del hemidiafragma derecho, presencia de derrame pleural de diversa magnitud y no se encontró compromiso de la cavidad pericárdica (Figura 2). La tomografía axial computada (TAC) se realizó sólo en cuatro casos: uno en quien se confirmó lesión neoplásica hepática y salió del estudio, uno por Ascaris lumbricoides y dos por AHNA (Figura 3). Se encontró AHA y AHNA tanto en el lóbulo derecho como en el izquierdo; los AHA fueron únicos o múltiples con un máximo de 4. En los AHNA se encontraron entre 1-3 y únicamente en 2 casos se detectó la presencia de numerosos abscesos.

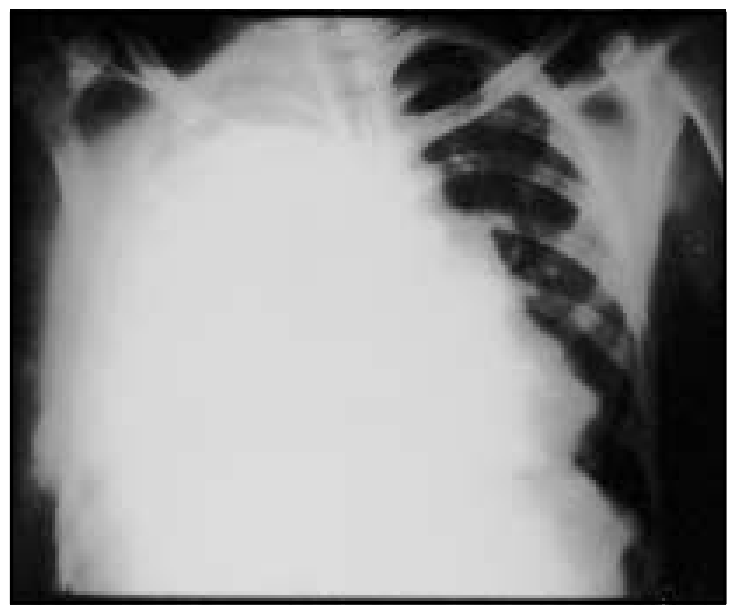

FIguRA 2. Radiografía de tórax con derrame pleural masivo. 


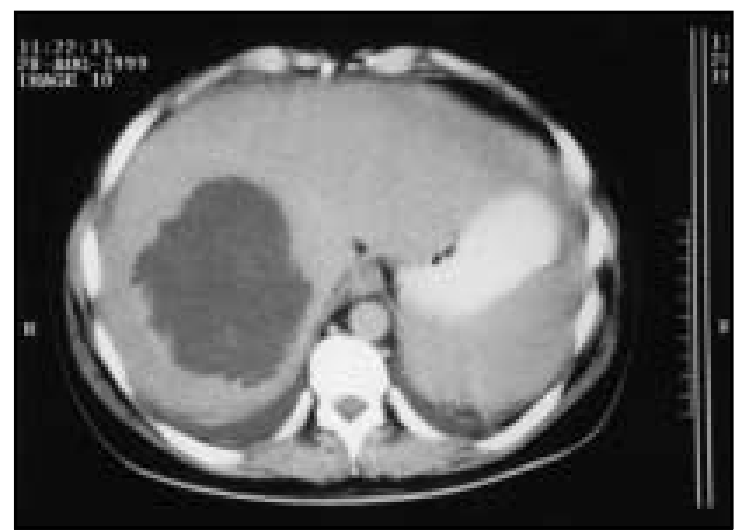

FIgURA 3. TAC con absceso hepático único.

En los casos de AHA las pruebas de ELISA fueron positivas en 100\% y las de ID en 93\%. Para el AHM, $100 \%$ de las pruebas fueron positivas. Los hemocultivos se lograron realizar en $42 / 49$ y fueron positivos para anaerobios en 5 pacientes de los cuales 4 tenían Peptococcus sp y 1 Propionibacterium sp. Los cultivos del drenaje hepático fuemon positivos en 7 pacientes, en 2 se aislaron varios gérmenes, distribuidos en anaerobios: 1 Bacteroides sp, 1 Fusobacterium sp y 1 Peptostreptococcus; anaerobios facultativos: 2 Staphylococcus coagulasa negativa, 2 Streptococcus no hemolítico del grupo D, 1 E coli, 1 Klebsiella sp, 1 Staphylococcus sp, 1 Staphylococcus aureus, 1 Streptococcus $\alpha$ hemolítico grupo D y 1 Streptococcus $ß$ hemolítico.
Sin importar la etiología, la complicación más frecuente fue el compromiso pulmonar así: elevación del hemidiafragma en 12 AHA, 6 AHNA y en los 4 con AH mixto; derrame pleural de diversa magnitud en 9 AHA, 6 AHNA y en los 4 con $\mathrm{AH}$ mixto. En segundo lugar, el drenaje a la cavidad peritoneal en dos casos de AHNA. Igualmente, la respuesta inflamatoria sistémica se presentó en todo tipo de $\mathrm{AH}$.

En cuanto al tratamiento los pacientes con AHA recibieron metronidazol oral 1,5 gr/día durante 10 días y se sugirió complementar con antiamebiano luminal al egreso. Se realizó punción hepática dirigida por ecografía a 7/29 pacientes con AHA y a 13/16 pacientes con AHNA, de los cuales 1 caso fue por Ascaris lumbricoides (un paciente con AHA y otro con AHNA presentaron ruptura a cavidad abdominal). A los pacientes de AHNA se les realizó punción hepática terapéutica y diagnóstica, excepto cuando presentaban AHNA pequeños, al mismo tiempo recibieron antibióticos como ampicilina-sulbactam, gentamicina y metronidazol en esquemas combinados. En el caso de Ascaris lumbricoides se dio antihelmíntico (Figura 4).

En la Tabla 1 se observan los resultados del análisis bivariado de las variables diligenciadas completamente en la base de datos. Se observan 5 variables que resultaron con un IC inferior a 95\% de área ROC $>0,5$. La Tabla 2 presenta el modelo final de la regresión binomial y las áreas $\mathrm{ROC}$ a

Tabla 1. Riesgos relativos del análisis bivariado y AROC de las variables

\begin{tabular}{|lcccc|}
\hline Variable & RR & IC 95\% & AROC & IC 95\% \\
\hline Dolor pleurítico & 1,48 & $0,97-2,24$ & 0,6468 & $0,5036-0,7833$ \\
Edad <40 años & 1,81 & $1,05-3,13$ & 0,6913 & $0,5458-0,8175$ \\
No presentar hepatomegalia & 1,57 & $1,10-2,23$ & 0,6648 & $0,5246-0,8005$ \\
Hematocrito $\geq 35 \%$ & 1,55 & $1,00-2,39$ & 0,6619 & $0,5036-0,7833$ \\
TP paciente - TP control $<1,5$ & 1,84 & $1,10-3,07$ & 0,7074 & $0,5673-0,8342$ \\
\hline
\end{tabular}

Tabla 2. Modelo final de la regresión binomial y AROC a medida que se incluyen las variables

\begin{tabular}{|lcccc|}
\hline Variable & RR & IC 95\% & AROC & IC 95\% \\
\hline TP paciente - TP control $<1,5$ & 1,65 & $1,03-2,65$ & 0,7074 & $0,5036-0,7833$ \\
Edad $<40$ años & 1,57 & $0,98-2,51$ & 0,8002 & $0,6566-0,8976$ \\
Hematocrito $\geq 35 \%$ & 1,24 & $0,91-1,69$ & 0,8504 & $0,7276-0,9406$ \\
\hline
\end{tabular}

Ho AROC 1=AROC 2=AROC 3; $\mathrm{p}=0,0061$ 
medida que se añaden variables en el modelo. Entre las 3 áreas hay una diferencia significativa $p=0,0061$, siendo el modelo con las 3 variables el que presenta mayor área. La adición de nuevas variables no aportó aumento al AROC de manera significativa. En la Tabla 3 se observa que presentar una elevación del PT menor de $1,5 \mathrm{~s}$ (TP paciente $-\mathrm{PT}$ control $=\mathrm{TPp}-\mathrm{TPc}$ ), ser menor de

Paciente con sospecha clínica de absceso hepático

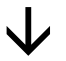

Exámenes de laboratorio: cuadro hemático, VSG, función hepática Imaginología: radiografía de tórax, ecografía hepática

Pruebas inmunodiagnósticas: ELISA IgG e inmunodifusión
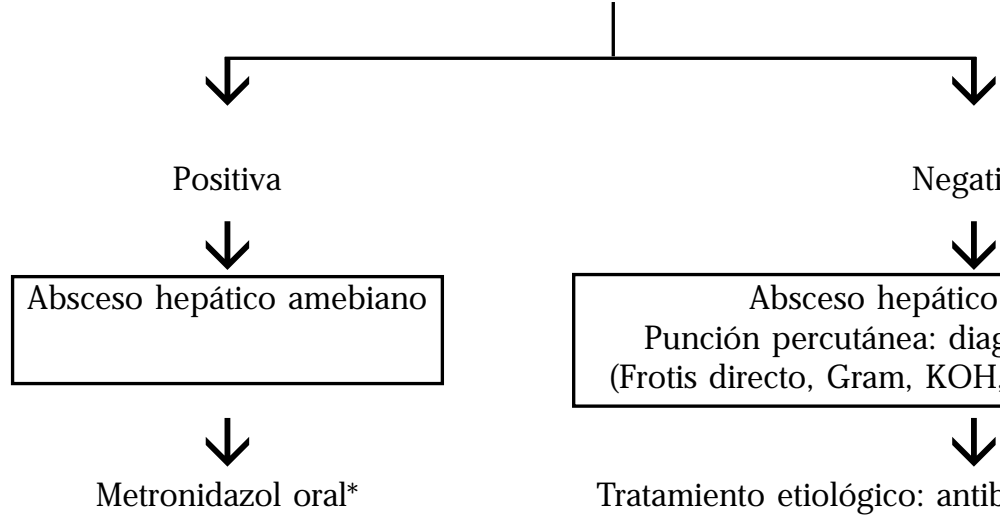

Negativa

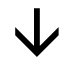

Absceso hepático no amebiano Punción percutánea: diagnóstica y terapéutica (Frotis directo, Gram, KOH, Ziehl-Neelsen, cultivo)

Tratamiento etiológico: antibióticos, antiparasitarios, antimicóticos

*Metronidazol IV en caso de intolerancia a la VO. Dosis $25 \mathrm{mg} / \mathrm{kg} /$ día durante 10 días. Adulto promedio de $60 \mathrm{~kg}$, dosis $1.500 \mathrm{mg} / \mathrm{día}^{22}$.

Figura 4. Flujograma de enfoque diagnóstico y terapéutico.

Tabla 3. Probabilidades y valor de las variables del modelo final para el diagnóstico de AH A

\begin{tabular}{|lcccccccc|}
\hline $\mathrm{p}$ & $\begin{array}{c}\text { AHA } \\
(\mathrm{n})\end{array}$ & $\begin{array}{c}\text { AHNA } \\
(\mathrm{n})\end{array}$ & TPp-TPc $<1,5$ & $<40$ años & $\mathrm{H} \geq 35 \%$ & $\mathrm{~S}$ & $\mathrm{E}$ & $\begin{array}{c}\text { Variable } \\
\text { positiva }\end{array}$ \\
\hline$\geq 0,31$ & 1 & 4 & 0 & 0 & 0 & 1,00 & 0,00 & \\
$\geq 0,39$ & 1 & 2 & 0 & 0 & 1 & 0,97 & 0,25 & $\mathrm{H}$ \\
$\geq 0,49$ & 3 & 3 & 0 & 1 & 0 & 0,94 & 0,38 & $\mathrm{E}$ \\
$\geq 0,51$ & 1 & 3 & 1 & 0 & 0 & 0,85 & 0,56 & $\mathrm{P}$ \\
$\geq 0,61$ & 4 & 2 & 0 & 1 & 1 & 0,81 & 0,75 & $\mathrm{E}+\mathrm{H}$ \\
$\geq 0,64$ & 5 & 1 & 1 & 0 & 1 & 0,70 & 0,88 & $\mathrm{P}+\mathrm{H}$ \\
$\geq 0,81$ & 7 & 1 & 1 & 1 & 0 & 0,55 & 0,94 & $\mathrm{P}+\mathrm{E}$ \\
$=1,00$ & 11 & 0 & 1 & 1 & 1 & 0,33 & 1,00 & $\mathrm{P}+\mathrm{E}+\mathrm{H}$ \\
\hline
\end{tabular}

0=negativo; $1=$ Positivo; $S=$ Sensibilidad; E= Especificidad; $H=$ hematocrito $>35$; $E=$ edad $<40$ años; $\mathrm{P}=\mathrm{TPp}-\mathrm{TPc}<1,5$ 


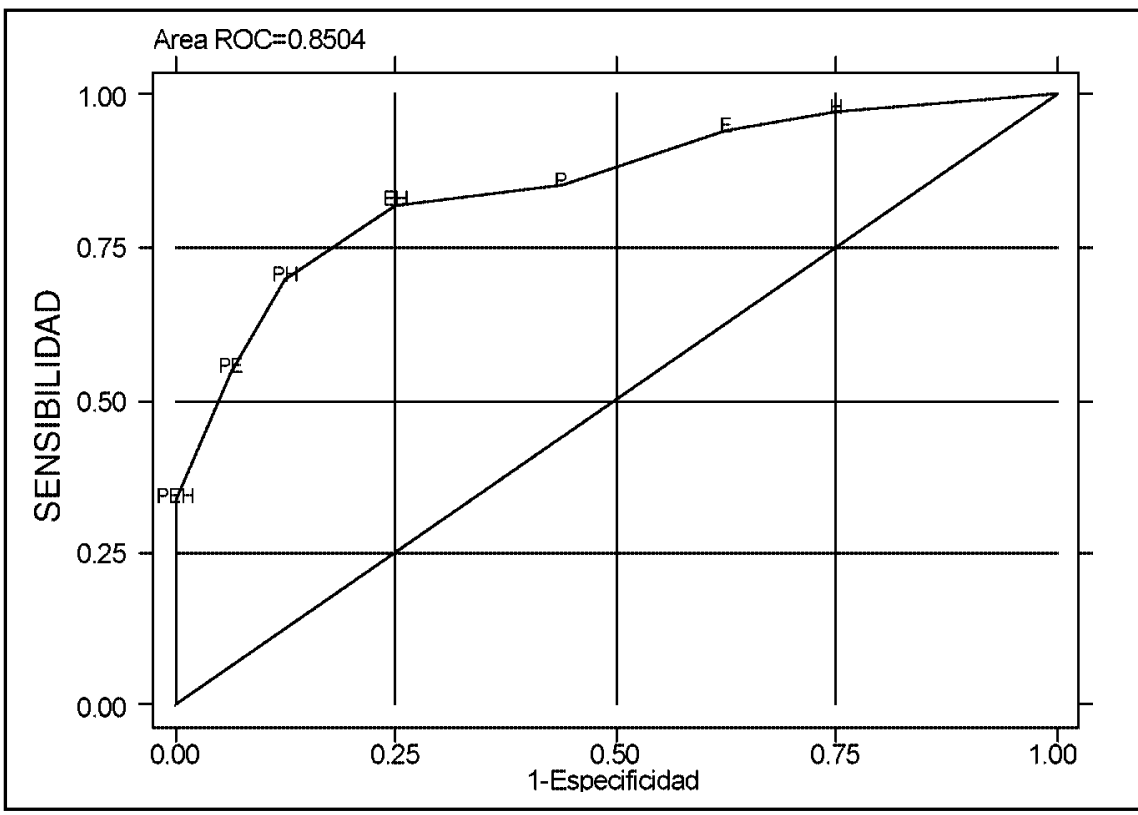

Figura 5. Area bajo la curva ROC del modelo final.

40 años y tener un hematocrito $\geq 35 \%$ da una sensibilidad de 0,33 y una especificidad de 1,00 para el diagnóstico de AHA. En la Figura 5 se observa la curva ROC del modelo final en donde se analiza que presentar 2 variables positivas ofrece una sensibilidad entre 0,55-0,81 y una especificidad entre 0,75-0,94.

\section{DisCUSIÓN}

En la literatura no se han definido criterios específicos del cuadro clínico, hallazgos de laboratorio, imaginología e inmunodiagnóstico para diferenciar el AHA del AHNA. Como lo han informado otros autores, los resultados obtenidos en este estudio mostraron que el AHA predominaba sobre el AHNA y que es más frecuente en hombres en edad productiva. En contraste, existen resultados reportados en otros estudios en áreas no tropicales donde la frecuencia del AHNA ha sido mayor que la del $\mathrm{AHA}^{13,14,23}$.

Se ha reportado una duración variable de la duración de la sintomatología, en los casos de AHA oscila desde una semana hasta meses, en esta investigación el rango de variación fue similar y estuvo entre 7-60 días ${ }^{12,14,23,24}$. Igualmente, la estancia hospitalaria fue prolongada en esta serie como ha sido reportada por Blanco y cols, con un promedio de 40 días ${ }^{12}$. En este estudio no se encontró diferencia estadística significativa en cuanto a los signos y síntomas, tampoco en los hallazgos del $\mathrm{CH}$ y el perfil hepático en AHA y AHNA, excepto por la presencia de dolor pleurítico en la base del hemitórax derecho para el AHA y la hepatomegalia con la prolongación del TP en el AHNA.

Por ecografía hepática o ultrasonografía (US) predominó el $\mathrm{AH}$ en el lóbulo derecho y en el izquierdo hubo $\mathrm{AH}$ de diversa etiología; en el caso del AHA se encontraron de 1-4 abscesos y en caso de AHNA de 1 hasta numerosos micro abscesos; el diámetro en los AHA varió desde 3-15,2 cm, con promedio de 8,3 $\mathrm{cm}$ y en AHNA de 4,3-14 $\mathrm{cm}$, con promedio de $9,1 \mathrm{~cm}$. La US es el método ideal no invasivo para el diagnóstico de $\mathrm{AH}$, puesto que es económico, no requiere inyecciones ni catéteres, y no produce radiación gamma ni rayos $\mathrm{X}$ por lo que puede ser usado en forma repetida incluso en mujeres embarazadas; sin embargo, es necesario tener en cuenta que de acuerdo al estado de evolución del absceso la ecogenicidad varía ${ }^{25,26}$. Es importante precisar que la US es dependiente de la experiencia del observador en contraste con 
la TAC, por tanto, si la US es negativa o dudosa y persiste la sospecha clínica de $\mathrm{AH}$ se debe continuar con una TAC dado su alta resolución que hace factible identificar lesiones de pocos milímetros de diámetro, en especial al usar medio de contraste. Por otra parte, la TAC muestra con gran precisión los órganos abdominales alrededor del hígado y tiene superioridad frente a la US para diferenciar lesiones intrahepáticas de extrahepáticas, también diferencia diversas lesiones focales del hígado como absceso, hemangioma, quiste y tumor. En conclusión, la imagen tomográfica a diferencia de la US tiene menor dependencia del operador; en consecuencia las indicaciones precisas para solicitar tomografía en $\mathrm{AH}$ son: diagnóstico no confirmado con otros métodos, persistencia de síntomas a pesar de tratamiento específico, diagnóstico diferencial con otras lesiones. En el presente estudio se realizó TAC cuando la ecografía no fue concluyente y hubo dificultades diagnósticas, llegándose a diagnóstico definitivo con ayuda de otros métodos: 1 caso de AH por Ascaris lumbricoides, dos pacientes que tenían múltiples abscesos piógenos (AHNA) y un caso que se excluyó del estudio por ecografía falsa positiva para AH que tenía lesión neoplásica. Por lo tanto, estas dos técnicas diagnósticas no permiten establecer un diagnóstico diferencial definitivo entre el AHA y el AHNA ${ }^{12,14,22,23}$.

El porcentaje de positividad de la prueba de ELISA IgG e ID fue similar al encontrado por otros autores $^{10,27,28}$. A pesar de la persistencia de títulos positivos de IgG por ELISA, contra E histolytica durante años en áreas endémicas, esta prueba continúa siendo la de referencia para buscar la etiología amebiana en $\mathrm{AH}$. Dado que ésta no puede diferenciar una infección aguda de una pasada en áreas de alta endemicidad, en este estudio se correlacionó la historia clínica y los resultados de laboratorio para definir el diagnóstico y tratamiento, quedando 4 pacientes con el diagnóstico de AHM por presentar ID, ELISA y cultivos positivos ${ }^{10,29}$; entonces, surge la pregunta de si alguno(s) de estos pacientes no tenía(n) AHM sino AHNA y memoria inmunológica para $\mathrm{E}$ histolytica. Por esto se requiere el desarrollo de nuevas pruebas diagnósticas que pueden diferenciar infección reciente de pasada por E histolytica y que, a su vez, tengan aplicación en la práctica clínica $^{30,31}$.
En los hemocultivos se aislaron gérmenes similares a los reportados en diversas series, anaerobios como Peptococcus sp, Propionibacterium sp. Los cultivos del drenaje hepático fueron positivos para anaerobios: Bacteroides sp, Fusobacterium sp y Peptostreptococcus, y anaerobios facultativos: Staphylococcus coagulasa negativa, Streptococcus no hemolítico del grupo D, E coli, Klebsiella sp, Staphylococcus sp, Staphylococcus aureus, Streptococcus $\alpha$ hemolítico grupo D y Streptococcus ß hemolítico. Por tanto, es importante pensar siempre en la etiología por anaerobios, por lo que deben hacerse cultivos en medios específicos. La sensibilidad de los hemocultivos es menor a la encontrada por medio de los cultivos del drenaje del absceso. En caso de AHNA se recomienda realizar cultivos seriados para microorganismos aerobios y anaerobios, en este caso se debe buscar la fuente de infección, comenzando por la vía biliar y el abdomen o en su defecto proceder a analizar fuentes distantes como el endocardio o la cavidad oral ${ }^{12,22,33}$.

Como se observa en la Figura 4 el frotis directo y las tinciones de Gram, $\mathrm{KOH}$ y Ziehl-Neelsen permiten identificar el agente causal. En esta investigación se encontraron otras etiologías: un caso excluido por Mycobacterium tuberculosus y otro incluido por Ascaris lumbricoides. Por tanto, es importante plantear que el diagnóstico de $\mathrm{AH}$ se sospecha clínicamente pero que se requiere de exámenes: imaginológicos, inmunodiagnósticos, microbiológicos y directo para confirmar su etiología. En caso de $\mathrm{AH}$ de origen bacteriano el drenaje es parte del tratamiento, sirve para aislar y precisar el tipo de germen y dirigir la terapia antibiótica específica ${ }^{12-14,34}$.

En este estudio se logró disminuir el número de punciones hepáticas en AHA, sólo en 8/29 pacientes, con evolución satisfactoria al tratamiento oral de metronidazol en $100 \%$ de los casos, en contraste a las punciones realizadas en caso de AHNA que fueron 13/17. Cuando se realice drenaje percutáneo, debe ser precedido por el inicio de antibióticos que deberán continuarse luego del procedimiento. Algunos autores han planteado que en caso AHNA, la aspiración del absceso da mejor información bacteriológica que los hemocultivos y por tanto puede ayudar a un uso racional de antibióticos. El drenaje percutáneo puede no ser necesario en pacientes con abscesos 
piógenos relativamente pequeños (menor de 5 $\mathrm{cm}$ ), algunos pacientes del estudio respondieron a los antibióticos sin necesidad de drenaje ${ }^{35}$. La punción evacuadora cada vez tiene menos indicaciones, pues el inmunodiagnóstico ayuda a precisar la etiología; por ahora estaría indicada en abscesos localizados en el lóbulo izquierdo y que tengan vecindad al pericardio, anticuerpos para $\mathrm{E}$ histolytica negativa, evolución tórpida luego de 48-72 $\mathrm{h}$ de haber iniciado el nitromidazol como terapia específica de AHA.

Se pueden realizar procedimientos como toracentesis, toracotomía, pericardiocentesis, ventana pericárdica o laparotomía de acuerdo con las complicaciones ${ }^{12,14,36-40}$. La evolución de los pacientes fue satisfactoria, sólo falleció una paciente con absceso piógeno originado en la vía biliar. En cuanto a las complicaciones, la más frecuente sigue siendo la pleuritis y el derrame pleural derecho en AHA o AHNA, el drenaje a la cavidad peritoneal sólo en dos de AHNA, en esta serie ningún paciente requirió drenaje de pericardio. Lo anterior se explica por los avances de métodos diagnósticos y terapéuticos que hoy en día permiten agilizar el enfoque del paciente y mejorar el pronóstico ${ }^{10,25,26,34,40}$.

$\mathrm{El} \mathrm{AH}$ en general es una enfermedad grave que requiere diagnóstico temprano y tratamiento inmediato. El cuadro clínico tiene síntomas y signos similares en AHA, AHNA o AHM. En nuestro medio, el diagnóstico de $\mathrm{AH}$ siempre debe contemplarse ante la presencia de síndrome febril de origen desconocido. La ecografía hepática es el examen imaginológico de primera elección ante la sospecha de AH por historia clínica,

\section{REFERENCIAS}

1. WalSh JA. Problems in recognition and diagnosis of Amebiasis: estimation of the global magnitude of morbidity and mortality. Rev Inf Dis 1986; 8: 228-38.

2. Thompson JE, Forlenza S, Verma R. Amebic liver abscess: a therapeutic approach. Rev Inf Dis 1985; 7: 171-9.

3. Cáceres E, Castaño de Romero L, Estupiñán D, López MC, Páez S, Pinila CA, Santacruz MM. En: Corredor A, Arciniégas E, Hernández CA eds. Parasitis- pero no se requiere seguimiento ecográfico para definir la mejoría del paciente, ésta se hace por medio de los datos clínicos, síntomas y signos, y de otros paraclínicos como el CH y la VSG. Autores como Ravdin reportan que la persistencia de la cavidad hepática, en un paciente asintomático, es decir con respuesta clínica adecuada al tratamiento, no amerita controles ecográficos repetidos que incluso pueden conducir a la realización de procedimientos invasivos innecesarios (punción hepática).

Los resultados sugieren que ante el diagnóstico clínico de AH confirmado por ultrasonido, y en ausencia de pruebas de ELISA e ID, el análisis de estas 3 variables predictivas (edad, hematocrito y diferencia del PT) tiene suficiente capacidad discriminatoria y permite inferir cuando es AHA o AHNA para tomar la decisión diagnóstica y terapéutica como se encontró la capacidad discriminatoria $(\mathrm{AROC}=0,85)^{15-18}$.

En este estudio la edad de los pacientes con AHA, fue alrededor de los 40 años como ha sido reportado ${ }^{12,14}$. Además, el hematocrito y el PT son pruebas que valoran la presencia de anemia y de la alteración aguda de la función hepática respectivamente, también son exámenes de bajo costo, disponibles en centros y hospitales de complejidad diversa.

Dados los avances en el inmunodiagnóstico de AHA y este modelo de variables para discriminar el diagnóstico etiológico, el clínico podrá contar con éstas para agilizar el enfoque diagnóstico y terapéutico. Por tanto, se pueden disminuir la morbilidad, la mortalidad, los procedimientos innecesarios y la estancia hospitalaria.

mo Intestinal. Ministerio de Salud, Instituto Nacional de Salud. Santa Fé de Bogotá: Instituto Nacional de Salud, 2000; 67-68.

4. Guzmán CE, López MC, Reyes P, Gómez JE, Corredor A, Agudelo C. Diferenciación de Entamoeba histolytica y Entamoeba dispar en muestras de materia fecal por detección de adhesina de E histolytica mediante ELISA. Biomédica 2001; 21: 167-71.

5. Kessel JF, Lewis WP, Solomon MA, Kim H. Preliminary report on a hemagglutination test for Entamoeba. Proc Soc Exp Biol Med 1961; 106: 409-13. 
6. MADDISON SE. Characterization of Entamoeba histolytica antigen-antibody reaction by gel diffusion. Exp Parasitol 1965; 16: 224-35.

7. Kessel JF, Lewis WP, MoLna C, Turner JA. Indirect hemagglutination and complement fixation tests in amebiasis. Am J Trop Med Hyg 1965; 14: 540-9.

8. Samrejrongroj P, Tharavanij S. Assessment of validity of counter immunoelectrophoresis and ELISA in the routine diagnosis of amoebiasis. Southeast Asian J Trop Med Public Health 1985; 16: 365-70.

9. JEANES AL. Indirect fluorescent antibody test in diagnosis of hepatic amoebiasis. Br Med J 1966; 5501: 1464.

10. Nichols RS, Restrepo MI, Duque S, López MC, CORREDOR AA. Standardization and evaluation of ELISA for the serodiagnosis of Amoebic Liver Abscess. Mem Inst Oswaldo Cruz 1994; 89: 53-8.

11. Arguello-García R, Sánchez G MC, Garduño G, Valadez-Salazar A, Martínez-García MC, Muñoz O ET AL. Evaluation of on Inmunoblot methodology for the detection of relevant Entamoeba histolytica antigens by antibodies induced in human amebiosis. Arch Invest Med 1990; 21: 3-9.

12. Blanco F, Novelua B, Sánchez P, Sanz J. Estudio descriptivo de 39 casos de abscesos hepáticos de origen piógeno (33) y amebiano (6). Ann Med Int 1995; 12: 477-84.

13. Bandres M, Burstein E, Casa J, Verona R. Absceso hepático tuberculoso: descripción de un caso y discusión de la literatura. Rev Gastroenterol Perú 1994; 14: 233-37.

14. Pinila RA, Lastra GG, Camacho MG, Cuevas EM, Díaz CK, Duque MN. Absceso Hepático en el Hospital San Juan de Dios, Bogotá. En: Chalem F, Casasbuenas J. Actas XIV Curso Anual Departamento de Medicina Interna, Universidad Nacional de Colombia, Bogotá. Acta Med Colomb 1996; 25-41.

15. Orozco LC, Camargo D. Evaluación de tecnologías diagnósticas y tipos de muestreos. Biomédica 1997; 17: 321-4.

16. MASTER RN. Micobacteriology. In: Clinical Microbiology Procedures Handbook. Isenberg HD Editor. ASM Washington DC, 1992.

17. WaLCholder S. Binomial regression in GLM. Estimating risk ratios and risk differences. Am J Epidem 1986; 123: 174-84.

18. HANLEY JA, McNeil BJ. The meaning and use of the area under receiver operating characteristic (ROC) curve. Radiology 1982; 143: 29-36.
19. KRAEMER HC. Evaluating medical tests. Objective and quantitative guidelines. Newbury Park: Sage Publications, 1992.

20. Delong ER, Delong DM, Clarke-Pearson Dl. Comparing the areas under two or more correlated receiver operating curves: A nonparametric approach. Biometrics 1988; 44: 837-45.

21. Stata Corp. 1999 Stata Statistical Software: Release 6.0. College Station, TX USA: Stata Corporation.

22. Arguelo JM, Gómez VR. Amebiasis Hepática. En: De la Entamoeba Histolytica a la Enfermedad Amebiana. Santafé de Bogotá; Laboratorios Synthesis. División Gastrointestinal.

23. RutTgi A, Rithcher J. Abscesos hepáticos piógenos y amebianos. En: Clínicas Médicas de Traducción por Folch A Clin Med North Am. México. Interamericana S. A. 1989; 921-35.

24. Maltz G, Knauer CM. Amebic Liver Abscess: A 15 Years Experience. Am J Gastroenterology 1991; 86: 704-10.

25. Kimura K, Stoopen M, Reeder MM, Moncada R. Amebiasis: Modern Diagnostic Imaging With Pathological and Clinical Correlation. Semin Roentgenol 1997; 32: 250-75.

26. Stoopen ME, Kimura к. Capítulo 3 Absceso hepático amebiano, piógeno y fúngico. En: Stoopen ME, Kimura K y Ros PR, editores. Abdomen: Hígado, bazo, vías biliares, páncreas y peritoneo, Tomo II. Primera edición. Philadelphia: Lippincott Williams \& Wilkins, 1999; 29-45.

27. Restrepo MI, Restrepo Z, López C, Viluareal E, AguirRe A, Restrepo M. Diagnostic tests for amoebic liver abscess: comparison of enzyme-linked immunosorbent assay (ELSA) and Counter immunoelectrophoresis (CIE). Rev Soc Bras Med Trop 1996; 29: 27-32.

28. Sathar ma, Simjee AE, Nel JD, Bredenkamp BL, Gathiram V, JaCKSON TF. Evaluation of an enzymelinked immunosorbent assay in the serodiagnosis of amoebic liver abscess. S Afr Med J 1988; 74: 625-8.

29. Ravdin JI. State of the Art Clinical Article. Clin Infection Dis 1995; 20: 1453-66.

30. Abd-Alua M, El-Hawey AM, Ravdin JI. Use of an enzyme-linked immunosorbent assay to detect anti-adherence protein antibodies in sera of patients with invasive amebiasis in Cairo. Egypt Am J Trop Med Hyg 1992; 47: 800-4. 
31. Soong CJ, Kain KC, Abd-Alua M, Jackson TF, Ravdin JI. A recombinant cysteine-rich section of the Entamoeba histolytica galactose-inhibitable lectin is efficacious as a subunit vaccine in the gerbil model of amebic liver abscess. J Infect Dis 1995; 171: 645-51.

32. CRIPIN JS, Wang KK. An Unrecognized Etiology for Pyogenic Hepatic Abscesses in Normal Hosts: Dental Disease. The American Journal of Gastroenterology 1992; 7: 1740-3.

33. Pinila AE, López MC, Ricaurte O, CastiLo B, MurCia MI, Nichols RS et AL. Liver abscess caused by Ascaris lumbricoides: Case report. Rev Inst Med Trop S Paulo 2001; 43: 343-6.

34. RaLs PW. Focal inflammatory disease of the liver. Radiologic Clinics of North America 1998; 36: 377-89.
35. Huang CJ, Pitt HA, Lupsett PA, Osterman FA, Lujemoe KD, Cameron JL et al. Pyogenic Hepatic Abscess. Changing Trends Over 42 years. Ann Surg 1996; 223: 600-9.

36. Vareia E, HuRTado M, Fassier S. Absceso hepático amebiano y piógeno: pautas de comportamiento diagnóstico y terapéutica. Acta Med Colomb 1990; 15: 264.

37. Alvarez W, Cuello J, Trout G, González F, Acosta A, Cotes E. Absceso Hepático Amebiano. Acta Med Colomb 1992; 17: 110.

38. Petri WA, Singh U. State of the Art Clinical Article. Diagnosis and Management of Amebiasis. CID 1999; 29: 1117-24.

39. Marín E, Pinila AE, López MC. Absceso hepático amebiano. Revisión de 100 años de esta patología en Colombia. Acta Med Colomb 2000; 25: 218-26.

Agradecimientos

La División de Investigación de la Sede Bogotá de la Universidad Nacional de Colombia, por la financiación códigos 709150-809159. A los Drs. Augusto Corredor, Ernesto Marín, Gloria Pardo, Guido Lastra, Héctor Reverend, Iscay Ronderos, Jorge Armando Rojas, José Manuel Arboleda, Luis Heber Ulloa y Vladimir Corredor de la Universidad Nacional de Colombia y Adriana Arévalo del Instituto Nacional de Salud. 